\title{
MARGINACION E INADAPTACION (NOTAS PARA UN ESTUDIO SOBRE LA LLAMADA DELINCUENCIA JUVENIL EN BARCELONA)
}

\author{
Juli Sabaté \\ (Universidad de Barcelona)
}

La pretensión de sugerir unas notas para el tratamiento sociológico de la marginación social y una đe sus manifestaciones más evidentes (la llamada «delincuencia juveril»), desde una perspectiva autónoma y teniendo a Barcelona como telón de fondo, es el objetivo del presente artículo. En una primera parte se repasan las grandes corrientes teóricas que han estudiado los temas de la marginación social (localizadas fundamentalmente en USA y Latinoamérica); a partir de todo ello y con la incorporación de algún elemento original se intenta elaborar una síntesis útil. En la segunda parte se indica una posible aplicación de la síntesis al estudio de la llamada «delincuencia juvenil», proponiendo un modelo explicativo adecuado. Se examinan también las dificultades que se presentan al aplicarlo a la realidad social de Barcelona y se dan posibles líneas de solución a estas dificultades. 
Un fantasma tecorre Europa: la emergencia de los marginados/marginales (la llamada «inseguridad ciudadana», la rebelión de los jóvenes, el reto a la heterosexualidad, los movimientos de locos, presos, minusválidos, etc.). Parece como si la integrada Eutopa descubriera -al socaire de la crisis que la atenaza en los últimos años- profundos desgarrones en su, aparentemente perfecto, tejido social.

A todo esto, Barcelona (ciudad de ferias y congresos) no es ninguna excepción: ha vivido en los últimos veinte años una explosión hacia dentro (aumentando su densidad hasta límites alucinantes) y hacia fuera (creando un cinturón - llatuado rojo- que la atenaza por todas partes menos por una que da al mar), producida por un precario - por lo que se ha vistodesarrollo industrial y una especulación de agárrate-y-no-te-menees. El resultado meramente visual es un caos urbano objeto ya de estudio internacional con la degradación galopante de unas zonas, la segregación casi total de otras y —n general- la ausencia de «suavizadores» (por ejemplo, servicios colectivos de todo tipo). Por otra parte, Barcelona es políticoculturalmente un maravilloso ejemplo de esquizofrenia colectiva: está tocando a la mítica Europa (la «pequeña Europa» se la tlama - supongocon una cierta sorna), con lo que esto podría comportar de progresividad y tolerancia en sentimientos, actitudes y comportamientos. Pero la política, las leyes y por supuesto la cultura «oficiales» son tan hispánicas como en cualquier otro tincón de la piel de toro. Resultado: Barcelona es una ciudad (o un continuum urbano) con enormes zonas y/o barrios marginados ecológica, cívica y culturalmente. Esto es tan socialmente reconocido que en el lenguaje popular se habla ya de alguna de ellas como de "ciudad sin ley» y hasta se ha llegado a rodar en uno de ellos una ambigua película - por otra parte de éxito- sobre la llamada delincuencia juvenil. Esta película, al ser visionada en estos barrios, ha producido en el público adolescente fenómenos de identificación bastante significativos.

Por otra parte, Barcelona es una ciudad con un aumento de la inseguridad ciudadana tanto por el crecimiento de la delincuencia como por el 
creciente sentimiento colectivo de victimización —clave para las operaciones de la reacción social. Barcelona es también un foco lógico de movimientos reivindicativos de la «diferencia marginal» que encuentran un caldo de cultivo favorable a su existencia y al desarrollo de actividades organizadas, tanto cuantitativamente (por la eterna ley de las relaciones entre la unión y la fuerza) como cualitativamente (por la ya sugetida esquizofrenia). Así se han visto surgir y funcionar gestoras de minusválidos, coordinadoras de talleres de subnotmales, coordinadoras de jubilados, etc. $Y$, en fin, Barcelona es un oasis para los grupos de liberación sexual que contestan la heterosexualidad dominante y se reclaman con orgullo de su —actualmarginalidad. El hecho es que en Barcelona funcionan con una casi legalidad total diversos grupos homosexuales masculinos y femeninos (y esto al margen de las controversias que existen entre ellos y que refejan las contradicciones existentes en su seno).

La sociología taramente ha penetrado en este terteno de la marginación social y su actual emergencia y lo ha ido dejando o bien a otras reflexiones científicas (la psicología o el derecho) o a otras prácticas «menos desinteresadas» (la política, la caridad...). ${ }^{1}$ Parece evidente que se ha llegado a un ciesto punto a partir del cual empieza a ser necesario el análisis sociológico del asunto. Se impone, pues, ir discutiendo los pocos modelos explicativos que la sociología ha elaborado sobre la marginación social en su actual emergencia y, a partir de ahí, pensar en ir elaborando una sintesis nueva, etc.

Uno de los «puntos calientes» del tema es la llamada delincuencia juvenil: plaga - dicen- que azota nuestras ciudades y de la cual sólo tenemos una definición, la jurídica: delitos cometidos por menores de edad penal. Por ahí andaría el objeto de este artículo: sugetir unas «notas» para la discusión sociológica de la marginación social e intentar concretarlas en una aproximación a la realidad compleja y notoria (psico-jurídico-social como mínimo) de la llamada delincuencia juvenil, a ser posible en Barcelona.

\section{Discutiendo modelos}

A la sociología española nos han llegado básicamente dos grandes corrientes teóricas al respecto: una centrada en la diferencia y la otra preocupada por la marginalidad.

1. En el «Directori 1978» de l'Associació Catalana de Sociologia sóto constan tres individuos interesados en el tema de la marginación social de un total de 113. 
La primera se fue elaborando en USA desde la segunda década de nuestro siglo. Es, por tanto, debido a la considerable perspectiva que ya tiene y a los no menos considerables recursos utilizados, teóricamente potente y empíticamente notable. El rótulo que más aptoximadamente podría englobarla sería el de «conducta desviada», y su temática fundamental la búsqueda del por qué, el cómo y el cuándo de las conductas diferentes. Conviene resaltar que lo normal es que los norteamericanos -excepto algunos casos- no se hayan dedicado a estudiar la marginalidad, sino la diferencia. Ello es debido tanto a la originalidad de su estructura social e imperial como a causa del matco teórico de referencia casi absoluto de su sociología (individuo actor).

A pesar de la simplificación que ello comporta, puede ser útil sintetizar y clasificar en tres grandes líneas de estudio las aportaciones de la devion$c e$. En primer lugar, la centrada en la anomia (desajuste entre la estructura cultural y la estructura social) como situación productora de conductas desviadas. ${ }^{2}$ En segundo lugar, el conjunto de explicaciones especificamente orientadas al fenómeno de la delincuencia (y en especial su aprendizaje en la llamada delincuencia juvenil) que fueron surgiendo en la Universidad de Chicago a partir (teóricamente, que no cronológicamente) del planteamiento de la "asociación diferencial», y que pueden o no haberse conectado. con la temática de la anomia. ${ }^{3}$ Ejes fundamentales de este análisis fueron subcultura y bandas. ${ }^{4}$ Por último, una nueva pista para el estudio de la desviación la han ido dando los sociólogos implicados en la que se ha dado en llamar la «teoría del etiquetaje»; para éstos hay una cuestión definitiva en la carrera desviada de un individuo, que es el hecho de haber sido objeto de la reacción social —cierta o falsa- y haber sido etiquetado -a partir de ahí - como desviado.

Por supuesto que esta simple clasificación no puede agotar todos los matices de la deviance, pero nos coloca ante las tres líneas teóricas más importantes: anomia, subcultura y bandas, y etiquetaje. Las características comunes que podríamos excontrar en las tres pueden ser perfectamente

2. Robert K. Merton, Teoria y estructura sociales (México: Ed. FCE, 1964). Marshall Clinatd, Anomia y conducta desviada (Buenos Aires: Ed. Paidos, 1967), con una exhaustiva bibliografía sobre la anomia.

3. Edwin Sutherland, Principios de criminologia (París: Ed. Cujas, 1966).

4. Albert Cohen, Delinquent boys: the culture of the gang (Nueva York: The Free Press, 1955) y Richard Cloward y Lloyd Ohlin, Delinquency and opportunity: a theory of delinquent gangs (Nueva York: The Free Press, 1960).

5. Howard Becker, Los extraños, sociologia de la desviacion (Buenos Aires: Ed. Tiempo Contemporáneo, 1971) y Edwin Lemert, Social patbology (Nueva York: Mac Graw Fill, 1951). 
los puntos comunes a toda la sociología USA, una sensata percepción de la realidad y un enfogue centrado en el individuo y en las peripecias de su conducta.

La otra gran corriente teótica es más joven y también nos ha llegado de América, pero esta vez del Sur. Su rótulo puede ser la «marginalidad» y su estudio surgió cuando las poblaciones - marginales durante los últimos cuatro siglos- de Latinoamérica empezaban a hacer su aparición en la vida pública (década de los sesenta). Por lógica, el enfoque del Sur tenía que ser diferente al enfoque del Norte: lo importante no era la conducta desviada, sino la aparatosidad de la situación de marginalidad de enormes capas de la población que funcionaban totalmente al margen de la vida occidental.

En principio fue la marginalidad ecológica, y luego, a partir de la asimilación teórica de marginalidad a no participación, se estudiaron todas las demás: la ausencia de participación política, laboral, cultural, etc. Este análisis partió en un primer momento de unos presupuestos de tadicalidad y globalidad de la marginalidad debida a cuatro siglos de superposición cultural, para ir pasando a unos presupuestos de multidimensionalidad causada por la falta de absorción de la mano de obra por parte del sistema productivo de Latinoamérica. ${ }^{6}$ Siempre, no obstante, recalcaba que su emergencia era el resultado de los procesos de modernización y del llamado -eufemísticamente- contacto cultural.

Y bien. Así explica América sus cosas y esto es lo que nos ha llegado a nuestra sociología. Mas si España es diferente, debemos suponer que sus cosas también lo son. Por ello, surge la necesidad de it proponiendo algún modelo explicativo de nuestros desvíos y marginaciones, que (tomando todo lo que haya que tomar de América) tenga el sello de nuestra especificidad.

En este sentido, lo primero que se impone es considerar el lado diacrónico del asunto. Nuestra historia es una gloriosa crónica de moros y brujas, pícaros y bandoleros, vagos y maleantes. $\mathrm{X}$, si bien el marco temporal de estas notas queda reducido a la modernización que se opera en nuestro país en los últimos veinte años, es obligada la consideración de la crónica anterior para poder entender nuestra inercia colectiva. Vamos a ver, pues, cómo definimos nuestra tealidad, primero causalmente y luego descriptivamente.

6. Gino Germani, El concepto de marginalidad (Buenos Aires: Nueva Visión, 1973). Roger Veckemans, Marginalidad en América Latina, un ensayo de diagnóstico (Barceiona: Ed. Herder, 1969). José Nun, «Superpoblación relativa, ejército industrial de reserva y masa marginaly en Revisto Latinoamericans de Sociologia, 2 (1969): pp. 178-235. 
a) La etiología. Buscando las razones de las situaciones actuales de marginación social en España daremos con la palabra participación -por supuesto- y con la transformación de España en eso que Ilamamos una «sociedad de producción y consumo». Se supone que se margina a quien no participa de la producción y el consumo. Siguiendo por este hilo y preguntándonos por qué se margina a quien no participa, podemos formular la hipótesis de que si en nuestra sociedad de clase participación es igual a explotación (económica, política, cultural, etc.), se margina a quien no se puede explotat. Así, por ejemplo, se marginaría al subnormal porque no participa en el proceso productivo; es decir, porque no puede extraérsele plusvalía. Se marginatía a quien no participara de la heterosexualidad dominante $(o$, lo que viene a ser lo mismo, a quien no produce culturalmente los valores heterosexuales impuestos), porque no se le explota ideológicamente. El que no produce (económica o ideológicarnente) no es explotado, no es necesario, sobra, es marginado. Esta ausencia de participación no tiene que ser mitificadamente considerada como voluntaria. En una mayoría de ocasiones no sólo es involuntatia, sino incluso vivida negativamente.

Dentro de este complejo participación - explotación es evidente que hay que privilegiar de una forma notoria al área econòmica. La no participación económica produce la situación más visible de marginación social, la marginación ecológica, y acentúa las probabilidades marginadoras de la no participación en otras áreas de la vida social. Es de suponer que un análisis en profundidad de este punto podría permitir una útil aproximación etiológica al tema o, como mínimo, una útil discusión.

b) La descripción. El descubrir -a partir de abí- quiénes son marginados y cómo es la situación en que se manejan, pasaría por el análisis de un binomio fundamental; el compuesto por los elementos diferencia y etiqueta. También lo haría por su conexión a la etiología y al elemento determinante de ésta: la marginación económica y su más visible manifestación en la marginación ecológica.

Las combinaciones de los términos del binomio son múltiples. La diferencia puede ser una conducta -agresiva o no agresiva-, puede ser una situación - normalmente suftida-, y puede ser una alternativa buscada y asumida - activa o evasivamente. Por su parte, la etiqueta -la reacción social- puede ser más o menos contundente según la visibilidad o la peligrosidad que presente la diferencia. Puede crear identidad negativa

7. Además de quien no produce, queda también al margen el que produce lo que no hay que producir, sea económicamente (producción matginal: artesanía hippie, por ejemplo), sea ideologicamente (producir valores homosexuales). 
y, por ende, desviación secundaria. Incluso puede llegar a convertir a un no diferente en marginado (una conducta obediente percibida como falsa). ${ }^{8}$ Todas las posibles combinaciones de la diferencia con la etiqueta están a su vez sobredeterminadas por la variable marginación económica $\mathrm{y}$, en concreto, ecológica. A causa de esta multiplicidad de combinaciones, resulta siempre difícil el intento de describir clasiffcándolas las situaciones de marginación social. De todos modos - y a título de ejemplo- se pueden ofrecer algunas ideas.

Primero una clasiffación formulada a partir del elemento aparentemente más objetivo: la diferencia.

I. Conducta diferente. I.1. Agresiva (delincuentes de todo tipo).

I.2. No agresiva (prostitutas, toxicómanos...).

II. Situación diferente. II.1. Improductivos biológicamente o físicamente (ancianos, enfermos, minusválídos, subnormales; se podría proponer aquí al grupo de los niños).

II.2. Improductivos económicamente (para+ dos, lumpen...).

II.3. Improductivos culturalmente (minorías étnicas...).

II.4. Improductivos ideológicamente (homosexuales...).

III. Alternativa diferente. III.1. Activa (marginales políticos, religiosos, culturales...).

III.2. Evasivos (pasotas diversos...).

En segundo lugar, la etiqueta. Pero esto es ya más difícilmente objetivable. La reacción social no es uniforme $y$, en ella, se manifiestan las propias contradicciones de la sociedad de los «normales». En el fondo, detrás de cada sentimiento, actitud, gesto o comportamiento frente a la diferencia está la concepción que de cada "normal» tiene la sociedad, aunque la homogeneizacion ideológica es un hecho cierto modo grosso. Por tanto - y con las debidísimas precauciones- podríamos también proponer una clasificación aquí. Un cuadto de las estrategias frente a la diferencia que conducirán a diversas etiquetas y a diversos tipos de reacción social podría ser el siguiente:

8. Howard Becker, Los extraños, sociología de la desviación (Buenos Aires: Tiempo Contemporáneo, 1971), p. 27. 
1. Estrategia A. Trata de apartar-soportar (mecanismos de represión-caridad) la concepción «tradicional».

2. Estrategia B. Trata de buscar la rentabilidad social -si la hay-(rehabilitación y todos sus derivados: reinserción, etc.) de la concepción «neocapitalista».

3. Estrategia C. Trata de -mientras espera un horizonte de desaparición de la marginación en una futura sociedad socialista - buscar hoy la rentabilidad social — si la hay-. (rehabilitación y todos sus derivados: reinserción, etc.) de la concepción «socialista-teformista».

4. Estrategia D. Trata de considerar a los grupos marginados como úni$\cos$ revolucionarios hoy, reivindicando su diferencia marginal mediante la concepción «radical».

Desde estas estrategias y -como antes ya se ha insinuado- en función de lo visible o peligroso de la diferencia funcionará la etiqueta. Con ella, la reacción social graduará la institucionalización de su actuación (su estructura, sus dimensiones, sus reglamentos, etc.). Siempre al lado de cualquier combinación diferencia-etiqueta (complicando la descripción, pero - ioh paradoja! - clarificando la vida) está la marginación económicoecológica.

\section{Nuestro caso}

Probemos de aplicar este modelo propuesto a un caso concreto: la Ilamada delincuencia juvenil y su realidad - prevención y tratamiento- en Batcelona. Ante este hecho, de relativa novedad entre nosotros pero de larga tradición en otros países más centrales de nuestro mundo capitalista, habría que empezar intentando ver cuál es el modelo explicativo habitual. Para esto nos resulta suficiente de momento el análisis del mismo término «delincuencia juvenil».

Efectivamente, el término "delincuencia juvenil» hace teferencia fundamentalmente al aspecto jurídico (la delincuencia de los menores de edad penal) y conlleva, por tanto, una determinada reacción institucional (los tribunales de menores y los centros para la prevención y el tratamiento). Consiguientemente, podríamos avanzar la hipótesis de que el término «delincuencia juvenil» corresponde a un modelo esencialmente jurídico-institucional de este fenómeno social. Este marco teórico parece ser en exceso limitado para dar cuenta de todo el conjunto de elementos que componen las molestias que algunos chicos, socialmente muy determinados, ocasionan 
- pueden llegar a ocasionar a la sociedad «normal» y de la respuesta que ésta tiene ante ellos.

En cambio, desde nuestro modelo, es posible formular una explicación en términos del binomio marginador diferencia-etiqueta, capaz de desbordar el marco juxídico-institucional y de considerar la globalidad del pro. blema. Lógicamente, por ello, presenta mayores dificultades para su estudio.

Esta podría ser la explicación: la realidad de lo que llamamos «delincuencia juvenil» podría ser entendida como una combinación específica de los términos del binomio marginador condicionada por un marco habitual de marginalidad ecológica evidente y considerable. Sería, pues, una mezcla de situaciones diferentes y conducta diferente. Estas son motivadas por la improductividad biológica, por la pertenencia a un medio improductivo económicamente $o$ incluso por la pertenencia a un medio culturalmente diferente. Aquélla es consecuencia del carácter agresivo ya presente o futuro. Esta mezcla presenta la compleja realidad psicosocial de la inadapta. ción. Todo ello es reforzado con una etiquetación fácil por lo visible y lo peligroso -actual o en futuro previsible- de la diferencia, y con una consiguiente reacción social contundente, institucionalizadora y normalmente dirigida por las estrategias A y B.

Para acabar de perfilar esta explicación, habría que resaltar algunas cuestiones del elemento diferencia que nos permitan comprender la peculiaridad de la etiqueta:

1. La importancia del problema psicosocial de la inadaptación (fallo del proceso aprendizaje-integración-adaptación por deficiencia de las instituciones de socialización o por socialización diferente).

2. Lo evidente y peligroso de la «clase» de los chicos (utilizando el término «clase» para dos conceptos diferentes: la clase social y la clase -l tipo- de persona).

3. E1 fondo y la forma de las «molestias» que ocasionan o pueden llegar a ocasionar. El fondo porque los delitos juveniles más importantes numéricamente hablando son siempre el robo y el hurto y ambos atentan contra el núcleo fundamental de nuestra organización social que es la propiedad privada (aunque ellos no lo sepan). La forma tanto porque son unos menotes los que «molestan» a un mundo de adultos como por la espectacularidad de sus actos (bandas callejeras, navajas, tirones, etc.).

Resaltados estos tres elementos son más comprensibles la facilidad, contundencia y dureza de la teacción social que llegan hasta la prevención. En efecto, justamente porque hay que enseñar a respetar la propiedad 
privada y, al mismo tiempo, a «guardar las formas» a unos menores presumiblemente inadaptados de un medio ya de por sí marginal, es éste el. único caso en que la sociedad ha dispuesto un conjunto institucional para la prevención de una marginalidad y no solamente para su tratamiento posterior. Porque si la prevención de la matginalidad en cualquiera de sus formas no deja de ser hoy un bello deseo, ¿qué otra cosa son los centros. de la Junta de Protección de Menores y de otras instituciones religiosas. - laicas, públicas o privadas, como internados, casas de familia e incluso colectivos, sino centros de prevención de la llamada delincuencia juvenil, o -como a veces se dicem- centros de asistencia a chicos y chicas en «situación de predelincuencia»?

A continuación intentemos ver cuál es la situación en Barcelona. Resignémonos a confesar que no lo sabemos muy bien. Resulta difícil, hoy por hoy, conocer cuál es la realidad de la llamada delincuencia juvenil en Barcelona. Primero porque para operar en globalidad con nuestro modelo explicativo sería necesario -al margen de los posibles datos jurídico. institucionales conocidos- un trabajo de observación sistemático y pro. longado dentro de instituciones y fuera de ellas; en barrios marginales y segregados, y en barrios centrales e integrados; con los chicos y chicas y con sus medios. Un estudio así debería abordarse desde una perspectiva pluridisciplinar, trabajo que obviamente nadie puede de momento asumir.

Aunque ésta sea la situación, van en esta dirección algunas experiencias que hoy ya funcionan: los «educadores en bartios», los "casals cívics», los esfuerzos de algunas instituciones, como por ejemplo los «collectius. infantils de l'Ajuntament de Barcelona», la descentralización y colaboración con los bartios que presenta el new-look de la Guardia Urbana, e incluso la colaboración de la Universidad a través de grupos de estudiantes, para. estudiantes y postestudiantes que voluntariosa y esforzadamente trabajan en ello. Un ejemplo sería el Grup de recerques sobre la delinqüència juvenil incardinado al Departamento de Psicología Evolutiva de la Fa. cultad de Filosofía y Ciencias de la Educación, organizador de las primeras jornadas sobre la "prevenció $i$ el tractament de lo delinqüència juvenil a Catalunyas en diciembre de 1978 . No hay todavía perspectiva suficiente como para saber en qué pararán todos estos esfuerzos, pero podrían permitir aproximaciones útiles y provechosas a la cuestión estudio globalizador y pluridisciplinar del problema.

En segundo lugar, también es difícil operar con el modelo jurídico. institucional. Faltan datos suficientes desde esta óptica para analizar la llamada delincuencia juvenil en Barcelona. De momento, sólo contamos con: 1. Los datos del Tribunal Tutelar de Menores de Barcelona y de la Junta de Protección de Menores, que son las instituciones oficiales dependientes 
del Ministerio de Justicia. 2. Un estudio sobre "Las instituciones tutelares de menores" realizado por el Grup d'estudis sobre els marginats socials (GEMS) a lo largo de todo el curso 1976"1977 -todavía inédito e incluso en aigún punto por acabar- que pretendía «conèixer d'una forma sistematica els centres per a menors inadaptats dins una zona geogràtica ben determinada $i$ que correspon a l'àrea d'influència de Barcelona». ${ }^{9} 3$. Análisis desperdigados, concretos y muy coyunturales sobre instituciones determinadas (hechos sobre todo en ocasión de aniversatios o en momentos de conflicto institucional). ${ }^{10} 4$. El estudio en curso encargado por el director general de promoción social de la Conselieria de Governació de la Generalitat de Catalunya al Instituto de Reinserción Social (IRES) y definido por el director general así: «Actualmente se está elaborando un inventario sobre los recursos problemas y los recursos existentes en el terreno de la marginación y de la reinserción social en nuestro país. Nunca se había Hevado a cabo una tarea asî́ $[\ldots]$ nadie había estudiado realmente lo que existe en Cataluña en materia de instituciones públicas o privadas que se dedican al tratamiento de la marginación y de la reinserción. $\gg^{\text {"l }}$ Nos consta que los centros de prevención y tratamiento de la llamada delincuencia juvenil entran en este inventario.

Como prueba de la dificultad señalada, vamos a aportar solamente los dos bloques de datos de mayor amplitud y de más fácil accesibilidad. Unos de procedencia oficial (los del Tribunal Tutelar de Menores de Barcelona) y los otros todavía inéditos, sacados del estudio sobre «Las instituciones tutelares de menores» del GEMS. Ambos presentan la misma característica de ser necesarios, pero no suficientes: son precatios, escasos y de difícil operabilidad. Por otra parte son, hoy por hoy, incorrelacionables. La «corre. lacionalidad" de los dos bloques de datos sería lo más importante para empezar a globalizar aunque sólo fuera al primer nivel del modelo jurídicoinstitucional.

Los datos del Tribunal Tutelar de Menores de Barcelona hacen refe. rencia solamente a:

9. Perspectiva Escolar, 18 (1977), p. 20. En este número de la revista (pp. 20. 23) apareció un avance de este estudio cuando aún se hallaba en curso: los datos son, pues, incompletos. Los datos definitivos están inéditos, exceptuando los citados en este artículo.

10. Algín ejemplo podría ser: Equip d'Educadors de «Les Torres»: «Informe 77, protecció de menors» (documento policopiado, Lliçà d'Avall, 1977). "Informe sobre el Instituto Ramon Albó. Protección de Menores» (documento policopiado, Barcelona, 1976).

11. La Vanguardia Española (Barcelona, 30 de mayo de 1979). 
a) Número de expedientes y su clasificación (Tabla 1).

$$
\text { TABLA } 1
$$

Total de expedientes

Pendientes del año anterior ........ 3.045

Abiertos durante el año

Reabiertos durante el año

919

Fallados durante el año

Pendientes al final del año

b) Clasificación según las dos facultades del Tribunal: reformadora y protectora, de los expedientes abiertos o reabiertos durante el año (Tabla 2).

\section{TABLA 2}

Clasificación según tipos de expediente

\begin{tabular}{|c|c|c|c|}
\hline & Niños & Niñas & Total \\
\hline Facultad reformadora: delitos. & 1.821 & 77 & 1.898 \\
\hline Facultad reformadora: faltas. & 454 & 66 & 520 \\
\hline Facultad protectora...$\ldots \ldots \ldots$ & 140 & 113 & 253 \\
\hline$\ldots \ldots \ldots \ldots \ldots \ldots \ldots \ldots$ & 2.415 & 256 & 2.671 \\
\hline
\end{tabular}

c) Una elemental constatación de Ias circunstancias familiares, teniendo en cuenta únicamente si el menor vive en familia propia, ajena o sin familia (Tabla 3). 
«Papers»: Revista de Sociologia

TABLA 3

Circunstancias familiares

Viven con su familia ................ 2.502

Viven con familia ajena .............. 36

Viven solos .......................... 10

«No consta su situación» ............ 123

TotAL ........................... 2.671

d) Origen geográfico de los menores, indicando sólo la provincia de nacimiento (Tabla 4).

TABLA 4 *

Clasificación según su origen

\begin{tabular}{|c|c|}
\hline Barcelona ciudad ... & 979 \\
\hline Barcelona provincia ... & 467 \\
\hline 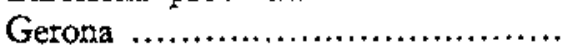 & 18 \\
\hline Lérida $\ldots \ldots \ldots \ldots \ldots \ldots \ldots \ldots \ldots \ldots \ldots \ldots \ldots \ldots \ldots$ & 10 \\
\hline 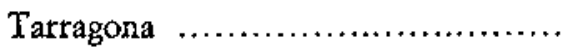 & 8 \\
\hline Fuera de Cataluña...$\ldots \ldots \ldots \ldots \ldots \ldots$ & 1.189 \\
\hline TOTAL $\ldots$ & 2.671 \\
\hline
\end{tabular}

* Este dato al referirse sólo al menor impide apreciar correctamente la variable emigración.

e) División por edad y sexo: la inmensa mayoría de menores que pasaron por el Tribunal fueron niños (2.415). El número de expedientes aumenta con la edad.

f) Motivos de enjuiciamiento: ocupa un primerísimo lugar en los motivos de enjuiciamiento todo lo relativo a atentados contra la propiedad privada. Del total de expedientes (2.671) un $66 \%$ son robos y hurtos en cualquiera de sus modalidades (1.780).

Si sólo contemplamos la actividad de la facultad reformadora, el porcentaje aumenta considerablemente. De los 1.821 expedientes un $85 \%$ corresponden a hurtos y robos (1.557). 
En la facultad reformadora el número total de expedientes fue el que se indica en la Tabla 5.

\section{TABLA 5}

Total de expedientes de la actividad reformadora

Pendientes del afio anterior

2.622

Abiertos durante el año .............. 1.510

Reabiertos durante el año ........... 908

Fallados durante el año .............. 2.095

Pendientes a final de año .......... 2.945

Los acuerdos iniciales tomados en los 2.095 expedientes fallados durante el af̃o fueron .los que se indican en la Tabla 6.

TABLA 6

Clasificación de acuerdos iniciales

Sin medidas:

Por inhibición en favor de otros TTM ....

Por inhibición en favor de otras jurisdicciones

Archivados provisionalmente

Otros

Medidas aisiadas:

Amonestaciones

Breve internamiento

Medidas duraderas:

Libertad vigilada

Asignados a una familia

Internados en establecimientos de educación o teforma

Internados en establecimiento de menores anormales 
Sumando a los 69 menores «internados en establecimientos de educación o reforma» por acuerdos del año 1976, el resto de menores que a final del año estaban bajo tutela del Tribunal Tutelar con medidas duraderas, ésta sería la situación:

$\begin{array}{lr}\text { Libertad vigilada ............................................ } & 64 \\ \text { Colocación en familia ..................................... } & 30 \\ \text { En casas de familia ................................... } & 20 \\ \text { Internados en establecimientos de observación ........ } & 220 \\ \text { Internados en establecimientos de educación y reforma. } & 274 \\ \text { Internados en establecimientos de menores anormales. } & 4\end{array}$

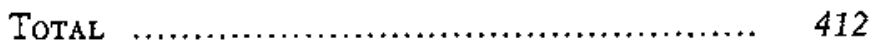

De los 1.510 expedientes abiertos durante el año en la facultad reformadora, éstos fueron los motivos de actuación:

646 por inhibición de las autoridades judiciales.

818 a requerimiento de autoridades gubernamentales.

31 a requerimiento de familiares.

2 a requerimiento de la Junta de Protección de Menores.

6 por denuncia de las víctimas, sus familiares o terceros.

1 por presentación espontánea del autor.

6 de offio.

\subsection{0 expedientes abiertos en total.}

EI Tribunal Tutelar de Barcelona es con mucho el que más trabajo tiene de España, llegando incluso a doblar en número de expedientes al segundo, que es el de Madtid.

De momento esto es lo que puede dar de sí un estudio de las cuestiones más significativas de la actuación del Tribunal Tutelar de Menores de Barcelona, con los datos que están a disposición del público. Los ełementos relevantes para una aproximación sociológicamente útil son evidentemente escasos.

Que Barcelona sea la ciudad más delincuenciada de España y que presumiblemente la emigración constituya un factor delictógeno de considerable importancia; que los niños cometan más delitos y faltas que las 
niñas; que el tipo de delito o falta más importante numéricamente sea el que atenta contra la propiedad privada; que el Tribunal Tutelar de Menores como única institución oficial sea relativamente impotente -de 2.095 acuerdos, 1.322 amonestaciones y 681 expedientes sin medidas - no dejan de ser generalidades del dominio público. Además, todos estos datos están desconexionados: no hay cortelaciones entre ellos. Pero lo más grave es que no hay relación con datos presumiblemente significativos para el sociólogo: medio familiar, vecinal, escolar, situación laboral, etc. Parece próxima, de todas formas, la publicación de un estudio sobre estas relaciones que se está realizando en la cátedra de Derecho Penal de la UCB.

El segundo y último bloque de datos sobre la realidad jurídico-institucional de Barcelona lo aporta el estudio del GEMS referido a instituciones tutelares de menores. En este trabajo están englobadas las instituciones de tratamiento (reformatorios y algunas casas de familias) y las de prevención (internados y ottas casas de familia). Según estos datos -inéditos todavia- habria en Barcelona detectadas un total de 69 instituciones: 46 internados y reformatorios y 23 casas de familia. La población atendida en estas 69 instituciones sería de 5.477 chicos y chicas; en los internados y reformatorios habría 4.876 chicos/chicas y en las casas de familia los 601 chicos/chicas restantes.

Los internados y reformatorios suelen ser edificios enteramente destinados ad boc - muchas veces son antiguas torres residenciales situadas en las afueras de Barcelona. Muy pocos son mixtos y los que lo son acogen a niños y niñas muy pequeños. La mayoría están regentados por religiosos o religiosas. Las causas de ingreso son diferentes: en los internados los motivos van desde la mera insuficiencia económica hasta el abandono; en los xeformatorios la entrada suele ser debida a alguna actuación «asocial» del menor.

De los 46 internados $\mathrm{y}$ reformatorios detectados:

Por otra parte:
18 tienen menos de 50 chicos/chicas.

9 tienen de 51 a 100 chicos/chicas.

19 tienen más de 100 chicos/chicas.

20 (casi la mitad) son religiosos.

4 dependen del Ministerio de Trabajo.

2 dependen directamente del Tribunal Tutelar.

6 dependen de la Junta de Protección de Menores.

4 dependen de la Diputación.

3 dependen del Ayuntamiento.

7 son centros privados llevados por seglares. 
Las casas de familia están situadas en el contexto urbano, normalmente en pisos. Su población de promedio es 15 chicos/chicas también habitualmente del mismo sexo. Se podrían distinguir dos tipos de casas de familia: en uno, la casa de familia es considerada como una prolongación del internado para aquellos chicos/chicas que una vez fuera del internado no pueden regresar a su familia por las razones que sea; en el otro, la casa de familia es considerada como una alternativa a las grandes instituciones. De las 23 detectadas: 16 tienen menos de 20 chicos/chicas y sólo 7 pasan de esta cantidad. Únicamente 5 son religiosas (parece que los religiosos/religiosas prefieren las grandes instituciones) y la dependencia institucional del resto es múltiple y variada, predominando la iniciativa privada - sea considerada como alternativa o no.

Una primera valoración de estos datos es la siguiente. ${ }^{12}$ Hay una desvinculación efectiva en la mayoría de casos de los organismos administrativos (Tribunal, Junta de Protección, Ayuntamiento...) con respecto a las instituciones que las convertía en auténticos «reinos de taifas». Existe un bajo nivel de preparación del personal encargado de las instituciones: falta de profesionalidad y mucha "vocación». Persiste una mentalidad muy benéfica en la mayoría de instituciones. Se produce una inexistencia de relación y de coordinación entre las diversas instituciones. Se provoca la masificación, la despersonalización y la segregación de los menores atendidos. Se constata una escasa relación con las familias, despreocupación por la problemática causante del ingreso en la institución. Se da una ausencia de planteamientos sobre las futuras salidas personales y profesionales de los menores atendidos. $Y$, en último lugar, hay una cuestión que luego volveremos a nombrar: la necesidad de crear en Barcelona un centro de observación y clasificación de menores.

La velocidad de nuestra vida político-social obliga, evidentemente, a considerar que los datos de este estudio en cuanto a número de instituciones y de menores atendidos en ellas puede haber ido variando en los últimos dos años. Pero al mismo tiempo está empezando a poner en peligro de obsolescencia la valoración de este estudio.

A nivel de la valoración es fácilmente apreciable que la dinámica institucional de hace solamente dos años era básicamente la correspondiente a una reacción social monodirigida por la estrategia A. Mientras en la actualidad podemos apreciar un lento pero parece que seguro cambio estratégico. Podríamos afirmar que la reacción social a nivel institucional está transicionando hacia unos presupuestos de rentabilidad social más acordes con la estrategia B (o como mínimo a una mezcla de A y B). Esta

12. Perspectiva Escolar, 18 (1977), p. 23. 
afirmación puede verificarse en los cambios operados en la estructura de las instituciones (por ejemplo, los grandes internados empiezan a caer en desgracia: uno ha cerrado definitivamente sus puertas y otro ha cambiado totalmente sus presupuestos socio-pedagógicos y su estructura) y en sus dinámicas (por ejemplo, el personal empieza a hacer cursos de preparación o de reciclaje, empieza a haber relación y una mínima coordinación, etc.).

Esto es todo lo que puede dar de sí de momento el conocimiento del mundo jurídico-institucional de la llamada delincuencia juvenil en Barcelona. Pretender ir hoy más allá (por ejemplo, cualificando con precisión todos estos datos) resulta bastante imposible. No podemos ni relacionar con exactitud los menores del Tribunal con los de las instituciones. No tenemos datos ciertos sobre el origen de clase (medio escolar, vecinal, escolar...), aunque todo el mundo (educadores, criminólogos, psicólogos, abogados, sociólogos, etc.) coincide en afirmar que la inmensa mayoría de casos del Tribunal Tutelar y de menores «institucionalizados» son de clase bajabaja. Y, por poner otro ejemplo de falta de cualificación, tampoco tenemos datos ciertos sobre la «gravedad» de los menores delincuentes, sobre su significatividad social o su intercambiabilidad. Éstos son los datos que hay y sus limitaciones.

Para operar con nuestro modelo es indiscutible que hay que empezar pluridisciplinariamente por ahí: verificar, actualizar, completar, relacionar estos datos. Luego, siguiendo con nuestro modelo, quedarían dos grandes tareas a realizar, una teórica y otra práctica.

La teórica pasaría por buscar las connotaciones personales y sociales de los menores institucionalizados o «tribunalizados» para ver su real significatividad (su «excepcionalidad» o su «normalidad»: «vivimos sobre verdaderos polvorines sociales, en esta ciudad hay doscientos mil niños en la calle que respiran un clima de predelincuencia», ${ }^{13}$ estudiando las aportaciones de todas las experiencias que hoy funcionan e imaginando nuevos terrenos de intervención teórica (estudio de las bandas, de la representación social, análisis de las instituciones, etc.), relacionándolo todo con el análisis de la estructura urbana del continuum barcelonés.

La práctica consistiría en la búsqueda de la lógica y necesaria coordinación entre las diversas experiencias e instituciones; y entre ellas y todos los «estudiosos» del tema. Otra cuestión sería la creación y puesta en marcha de un centro de investigación, observación y clasificación de los menores que asegurara efectivamente la coordinación, y de esta manera pudiera establecer las inevitablemente necesarias prioridades tanto teóricas como prácticas.

13. Antonio Figueruelo en Blanco y Negro (Madrid, 7 de febrero de 1978). 
Con todo esto se podría llegar a una real valoración cualitativa y cuantitativa de la «diferencia» y podría analizarse con mayor objetividad y verosimilitud la reacción social, la «etiqueta»; y al mismo tiempo se podría esclarecer el problema de la etiología.

$Y$ probemos de acabar con unos datos rápidos y elementales sobre una institución concreta, que bien podrían ser un ejemplo de por dónde y cómo se podría ir montando este análisis. La institución en cuestión es Els collectius infantils de I'Ajuntament de Barcelona, dependiente del Centre de Formació d'Educadors Especialitzats, que por su carácter de alternativa experimental puede ser util para cerrar estas notas. ${ }^{14} \mathrm{Els}$ collectius infantils de l'Ajuntament de Barcelona acoge a unos 400 chicos/chicas de 3 a 16 años en 8 colectivos desparramados por toda la geografía urbana de Barceiona y ubicados, preferentemente, en los barrios de origen (Born, Canyelles, Padró - barrio «xino», Poble Nou, Zona Franca, Poble Sec, Carmelo-Gràcia i Sants); cada colectivo -dividido en dos comunidades de 25 - tiene a su servicio un equipo de 8 educadores, una asistente social y un psicólogo a media jornada (el personal de los 8 equipos, sumados al de los «servicios generales», resulta en 83 personas).

Els collectius infantils de l'Ajuntament de Barcelona es uno de los dos grandes internados citados antes como ejemplos de la transición: ha pasado de religioso a seglar con vocación de servicio público; de ser un internado para chicos y otro para chicas en un extremo de la ciudad a funcionar en colectivos mixtos repartidos por la ciudad y por supuesto en pisos; de ser un internado reglamentado e «institución total» a ser una institución múltiple, abierta a las familias (tanto como es posible), abierta escolarmente, laboralmente, abierta a la ciudad y a los barrios, y preo. cupada pedagógicamente por la reestructuración de la personalidad del chico/chica a través de las intervenciones del educador en la vida cotidiana.

Evidentemente, por su importancia estratégica, hoy no es en absoluto una institución representativa ni a nivel de su funcionamiento y estructuta ni a nivel de la perspectiva socio-pedagógica (es una institución con tensiones constantes por la coexistencia en ella de prácticamente las cuatro estrategias de la «reacción social» antes mencionadas). Y justamente por todo esto hay que agradecer -o simplemente reconocer que es lógico que lo haga - que sea la única institución que hace públicos sus estudios, sus

14. El Centre de Formació d'Educadors Especializzats empezó a funcionar en 1969 adscritos a la división de formación permanente del ICE de la UAB: «El Centro de Formación de Educadores Especializados» (documento policopiado, Barcelona, Centro de Formación de Educadores Especializados, 1975). 
cuentas, sus debates internos, sus propias contradicciones, así como datos significativos sociológicamente como los siguientes: ${ }^{15}$

En un estudio global de la situación familiar de 355 chicos/chicas (correspondientes a 185 familias) y sobre un total de 71 barrios de origen (de Barcelona y de fuera de Barcelona) se puede apreciar que 6 barrios (La Mina, Besòs, Zona Franca, Born, Nau barris y barrio "xino»), identificables fácilmente como marginales, son el medio de origen de 175 chicos/ chicas. Según el mismo estudio, un $19 \%$ de los padres nacieron en Catalunya, un $70 \%$ fueta de Catalunya, y del resto $(11 \%)$ no se sabe. Un $49,1 \%$ de los padres son analfabetos o saben sólo «leer y escribir». Trabajan prácticamente la mitad de los padres (el $50,2 \%$ ), no trabajan un $19,7 \%$ y de los demás no se sabe. Finalmente un $22,7 \%$ de los padres tienen problemas de alcoholismo, 5 están en la cárcel y un 12,9\% de las madres se dedican a la prostitución. Ampliar, profundizar y sobre todo comparar estos datos podría ser un buen punto de partida.

15. «Els collectius infantils, memòtia curs 1977-1978" (documento policopiado, Barcelona, Centre de Formació d'Educadors Especialitzats, 1978). «Los colectivos infantiles, etapa de rodaje-teunión en Can Puig, perfeccionamiento organizativon (documento policopiado, Batcelona, Centre de Formació d'Educadors Especialitzats, 1978). «Los colectivos infantiles, etapa de rodaje - reunión en el Port. Los documentos iniciales» (documento policopiado, Barcelona, Centre de Formació d'Educadors Especialitzats, 1978). 